\title{
Stark width measurements of Fe II lines with wavelengths in the range $260-300 \mathrm{~nm}$
}

\author{
C Aragón ${ }^{1}$, P Vega $^{2}$ and J A Aguilera ${ }^{1}$ \\ ${ }^{1}$ Departamento de Física, Universidad Pública de Navarra, Campus de Arrosadía, E-31006 Pamplona, \\ Spain \\ ${ }^{2}$ Departamento de Física, Facultad de Ciencias, Universidad de Oviedo, C/Calvo Sotelo s/n, E-33007 \\ Oviedo, Spain \\ E-mail: carlos.aragon@unavarra.es
}

\begin{abstract}
The Stark widths of 21 Fe II lines with wavelengths in the range 260-300 nm have been measured using laser-induced plasmas as spectroscopic sources. A set of $\mathrm{Fe}-\mathrm{Cu}$ samples has been employed to generate the plasmas. To reduce self-absorption, each line has been measured using a different sample, with an iron concentration determined by means of the curve-of-growth methodology. The remaining error due to self-absorption has been estimated as lower than $10 \%$. Different instants of the plasma evolution, from $0.84 \mu$ s to $2.5 \mu$ s are included in the measurements. The electron density, in the range (1.6-7.3) $\times 10^{17} \mathrm{~cm}^{-3}$, is determined by the Stark broadening of the $\mathrm{H}_{\alpha}$ line. Within this range, the Stark widths are found to be proportional to the electron density. The Boltzmann plot method is used to obtain the plasma temperature, which is in the range 12900-15200 K. The Stark widths obtained have been compared with available experimental and theoretical data.
\end{abstract}

\section{Introduction}

The knowledge of Stark widths and shifts for atoms and ions is important for the diagnostics of laboratory and astrophysical plasmas. Also, the availability of experimental Stark broadening and shift parameters allows verifying calculations based on different schemes of atomic structure. In the case of Stark widths of ionized iron, the experimental and calculated data are scarce. Purić et al. [1] measured the Stark widths of fourteen Fe II lines from the resonance multiplets $a^{6} \mathrm{D}-\mathrm{z}^{6} \mathrm{D}^{0}$ and $\mathrm{a}^{6} \mathrm{D}-\mathrm{z}^{6} \mathrm{~F}^{0}$ using a low-pressure arc discharge. Dimitrijević [2] reported calculations of Stark broadening parameters for the Fe II multiplets $a^{6} \mathrm{D}-\mathrm{z}^{6} \mathrm{P}^{0}, a^{6} \mathrm{D}-\mathrm{z}^{6} \mathrm{D}^{0}$, and $a^{6} \mathrm{D}-\mathrm{z}^{6} \mathrm{~F}^{0}$. This lack of data for Stark widths of Fe II contrasts with the availability of transition probability data, compiled recently by Fuhr and Wiese [3].

In the last years, laser-induced plasmas have been used with increasing frequency as spectroscopic sources for the measurement of Stark widths. In the recent compilation article by Lesage [4], covering the period 2001-2007, laser-induced plasmas are included among the plasma sources for experimental determination of Stark widths and shifts. Works published later have reported measurements by laser-induced plasma spectroscopy of Stark widths of Ni II [5], $\mathrm{Zn}$ II [6], Sn I and Sn II [7], Pb II [8], and Mn I and Mn II [9]. One of the main requirements for accurate measurements of Stark widths is the existence of optically thin conditions, as selfabsorption leads to distortion of the line profiles and over-estimation of the line widths $[4,10]$. This condition is especially relevant for laser-índuced plasmas, which are characterized by high 
densities of atoms and ions. Generally, the Stark widths are measured using a single sample with a small content of the element of interest, in order to reduce self-absorption [5-7]. For example, in [5], an Al-Ni sample with nickel concentration of $2 \%$ was used to produce the plasma. A disadvantage of the use of a small content of the emitting element is the restriction of the measurement to lines of high or moderate intensity. In [8], a pure lead sample was used to measure the line widths of $\mathrm{Pb}$ II lines. By calculating the absorption coefficients of the studied lines, it was checked that the optical depths were low enough to consider them optically thin. However, for the most intense line studied, a tin-lead sample with $0.5 \%$ lead was used to reduce self-absorption after an estimation of the optical depth for different concentrations. In [9], a series of Fe-Mn samples was used to investigate the effect of self-absorption on the measurement of Stark widths of Mn I and Mn II lines emitted laser-induced plasmas. Singleand double-pulse configurations were compared. As self-absorption was much higher in the double pulse case, the Stark widths were finally determined in the single-pulse scheme.

In previous works, we have investigated the curve-of-growth methodology for the characterization of laser-induced plasmas, showing that the degree of self-absorption may be predicted for neutral atom and ion lines [11,12]. In the present work, we make use of this method to control self-absorption in the measurement of Stark widths of Fe II lines. The use of samples with different iron contents has allowed including in the study intense resonance lines as well as weak lines from multiplets of higher energy.

\section{Experimental setup}

A schematic diagram of the experimental setup is shown in figure 1. A Nd:YAG laser (wavelength $1064 \mathrm{~nm}$, pulse energy $100 \mathrm{~mJ}$, pulse width $4.5 \mathrm{~ns}$, repetition rate $20 \mathrm{~Hz}$ ) is focused at right angles to the sample surface by a lens of $126 \mathrm{~mm}$ focal length, the lens-to-sample distance being $116 \mathrm{~mm}$. The laser-induced plasma is generated in air at atmospheric pressure. A system of plane and concave mirrors is used to collect the plasma emission at right angles to the laser beam, forming a 1:1 image of the plasma on the entrance slit of a monochromator (CzernyTurner, focal length $0.75 \mathrm{~m}$, gratings of 1200 and 3600 lines $/ \mathrm{mm}$ ). The entrance slit of the monochromator was set at $20 \mu \mathrm{m}$ or $50 \mu \mathrm{m}$ depending on the spectral resolution requirements. The detector is a photomultiplier tube, whose signal is amplified and then captured by a digital oscilloscope. The Q-switch output signal is used to trigger the signal acquisition. The acquisition is programmed so that 500 samples of each signal, corresponding to different instants of the plasma evolution, are obtained in each laser shot. A home-made computer program controls the movement of the grating required for the desired wavelength scan. The different signals are stored in computer files, each one corresponding to the complete timedependent intensity of the plasma emission at a wavelength. In order to reduce the noise due to shot-to-shot fluctuations, nine acquisitions are averaged in each measurement. From the stored signals, the software obtains the spectra at selected time windows along the plasma lifetime.

In this experiment, nine iron-copper samples with iron contents in the range 0.5-25 wt. \% have been used. The samples have been prepared from the pure elements in powder form by pressing them to form pellets that have been melted in an induction furnace in argon atmosphere. The samples are finally grinded to obtain flat surfaces for plasma generation. During the measurements, the samples rotate at $100 \mathrm{rev} / \mathrm{min}$.

\section{Plasma characterization}

Six time windows with different delays from the laser pulse, centered at instants ranging from $0.84 \mu$ s to $3.6 \mu$ s have been selected for plasma characterization. The width of these windows increases with the delay, from $0.12 \mu$ s up to $1.4 \mu$ s. The widths were chosen small enough to 
reduce the variation of the plasma parameters within each time window, while keeping a noise level low enough for accurate measurements of the line profiles.

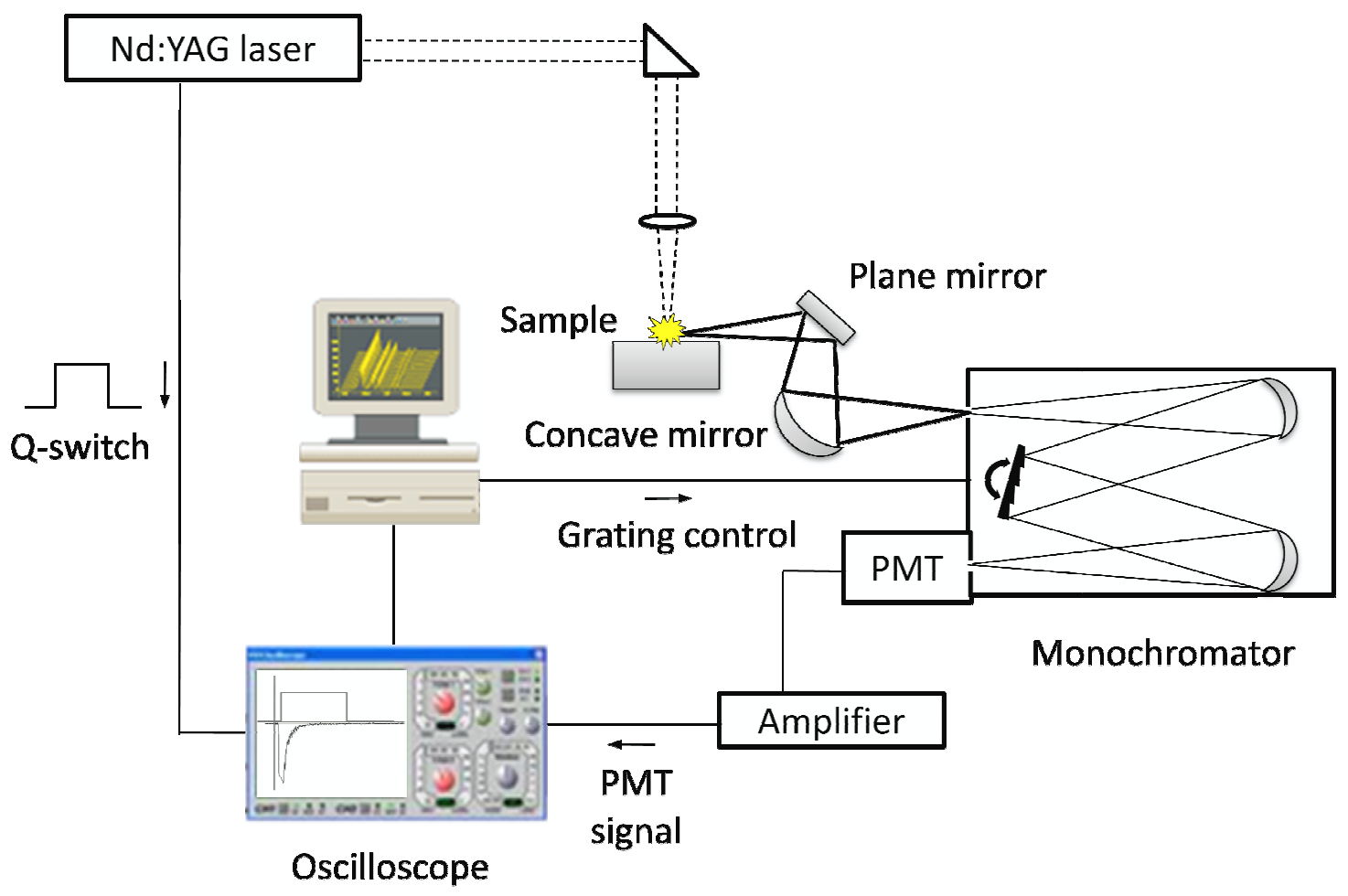

Figure 1. Experimental setup for the measurement of Stark widths.

\subsection{Electron density}

The electron density of the plasma at each time window was determined from the Stark broadening of the hydrogen $\mathrm{H}_{\alpha}$ line by using the diagnosis tables of Gigosos and Cardeñoso [13]. These tables provide the FWHM of the Lyman and Balmer series of hydrogen as a function of the electron density and the temperature. The spectrum of the $\mathrm{H}_{\alpha}$ line at $6562.79 \AA$ was measured using the grating of 1200 lines $/ \mathrm{mm}$ and a monochromator slit width of $50 \mu \mathrm{m}$. The instrumental width was determined as $0.56 \AA$ from the line spectrum emitted by a lowpressure neon lamp. The spectrum of the $\mathrm{H}_{\alpha}$ line for the time window centered at $1.3 \mu$ s is shown in figure 2. The FWHM of the line was determined by fitting the experimental spectrum to a Voigt profile, also shown in figure 2. All fittings to Voigt profiles in this work were carried out by means of a home-made least-squares fitting computer program. The Gaussian component of the Voigt profile was taken as the combination of the instrumental broadening and the Doppler broadening. The Lorentzian component of the fitting Voigt profile ranged from $9.9 \AA$ to $38.0 \AA$ for the selected time windows, and was assumed to be due to Stark broadening. 


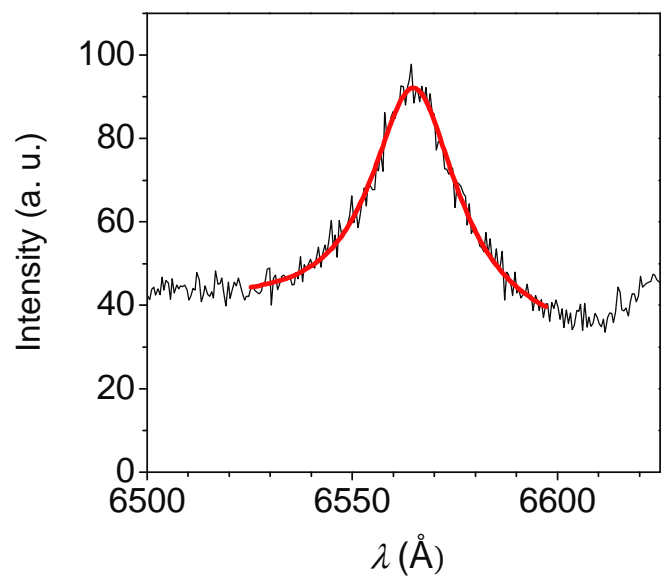

Figure 2. Spectrum of the $\mathrm{H}_{\alpha}$ line at $1.3 \mu$ s and fitting to a Voigt profile.

The electron density determined for different instants of the evolution of the laserinduced plasma is shown in figure 3. The error bars correspond to the statistical errors, determined by propagation of the standard deviations of the Lorentzian components of the fitting Voigt profiles. The results of figure 3 correspond to measurements made with the $\mathrm{Fe}-\mathrm{Cu}$ sample with an iron content of $3 \mathrm{wt}$. \%; measurements carried out with samples with other iron contents provided the same electron densities within the statistical error. The error of the electron density determined is mainly due to the error of the FWHM of the diagnosis tables used, which we have estimated as lower than $7 \%$ from the comparison of simulated and experimental results reported in table 4 of reference [13]. The propagation of this error leads to a relative error of the electron density of $11 \%$.

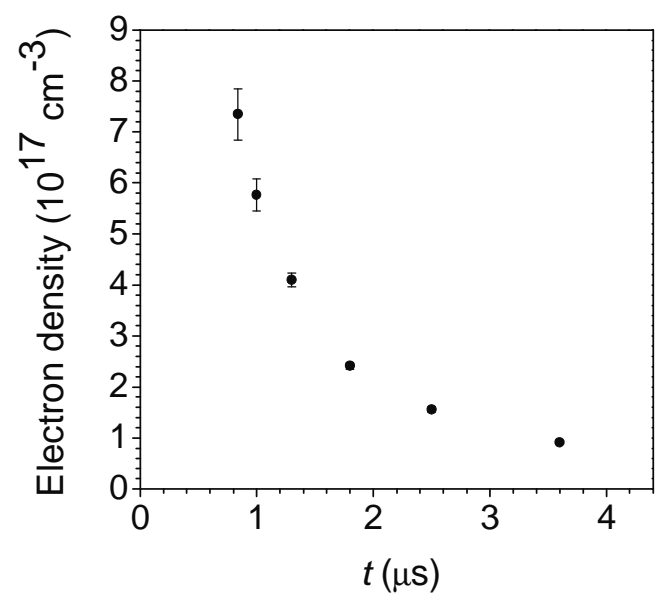

Figure 3. Electron density of the laser-induced plasma as a function of time elapsed from the laser pulse.

It should be noted that laser-induced plasmas are inhomogeneous sources, showing characteristic spatial distributions of the electron density and the other plasma parameters. In a previous work [14], we investigated the effect of this inhomogeneity on the measurements of the electron density by the Stark broadening of different reference lines. It was shown that, for a laser-induced plasma generated in similar conditions to the present one, the $\mathrm{H}_{\alpha}$ and the ion emissions come mainly from a central region of the plasma with nearly homogeneous electron 
density. As a consequence, the apparent electron density values determined in spatiallyintegrated measurements using the $\mathrm{H}_{\alpha}$ line and ion lines are compatible within the experimental errors. We may conclude that the $\mathrm{H}_{\alpha}$ is a good reference line to determine the electron density in the measurements of the Stark widths of Fe II lines, the error due to the spatial inhomogeneity of the plasma being negligible.

\subsection{Temperature}

The temperature of the plasma at each instant was determined by a Boltzmann plot constructed with seven Fe II lines, assuming local thermodynamic equilibrium (LTE). The LTE assumption is justified by the fulfillment of the McWhirter criterion [15], and also by the results of a previous work [16] where laser-induced plasmas in similar experimental conditions were characterized through Boltzmann and Saha-Boltzmann plots with high correlation to straight lines. In order to assure optically thin conditions in this measurement, the iron concentration in the sample was selected for each line by the procedure discussed below, in subsection 4.1. The line intensities normalized to the iron concentration were used to obtain the Boltzmann plots. A typical Boltzmann plot is shown in figure 4. The error of the temperature corresponds to the propagation of the error of the slope of the linear fitting. The temperatures obtained decreased from $15200 \mathrm{~K}$ at $0.84 \mu$ s to $11400 \mathrm{~K}$ at $3.6 \mu$ s. As mentioned before for the electron density, due to the plasma inhomogeneity, these values have to be considered apparent temperatures, resulting from the spatial integration of the emission coefficient of the ion lines, the emission at each point taking place at a different local electron temperature [16].

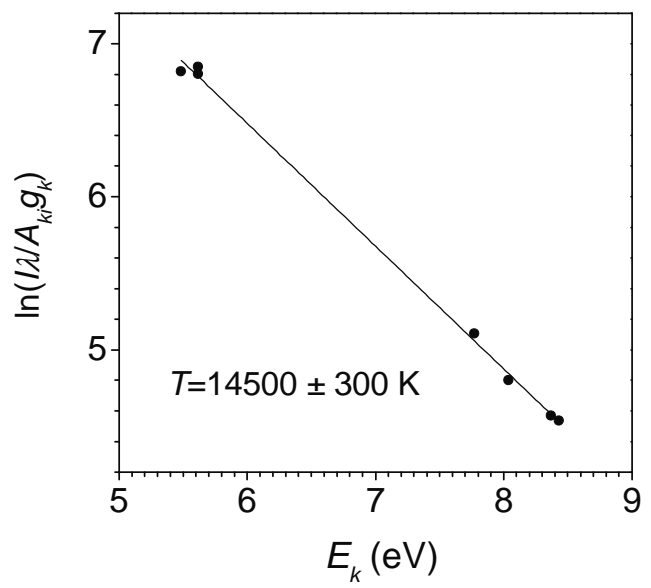

Figure 4. Boltzmann plot used to determine the temperature of the plasma at $1.3 \mu \mathrm{s}$.

\subsection{Curves of growth}

The characterization of the laser-induced plasma was completed by measurement and fitting of curves of growth, following the methodology described in our previous works $[11,12]$. The curves of growth, intensity versus concentration, were obtained for two Fe II lines using five Fe$\mathrm{Cu}$ samples with iron concentrations in the range 0.5-3.0 wt. \%. The curves of growth for the time window centered at $1.3 \mu$ s are shown in figure 5. If the plasma electron density and temperature values determined previously are used, the fitting of the experimental curves of growth to calculated curves provides two other parameters that complete the characterization of the plasma emission. In particular, the parameter $N^{\prime} l$, where $N^{\prime}$ is the atom number density for $100 \%$ concentration and $l$ is the length of the plasma along the line-of-sigth, is useful to obtain the self-absorption of the emission lines. The fitting curves are shown as solid lines in figure 5. 
The resulting values of the parameter $N^{\prime} l$ decreased from $8.1 \times 10^{20} \mathrm{~m}^{-2}$ at $0.84 \mu$ s to $2.8 \times 10^{20} \mathrm{~m}^{-}$ 2 at $3.6 \mu \mathrm{s}$.

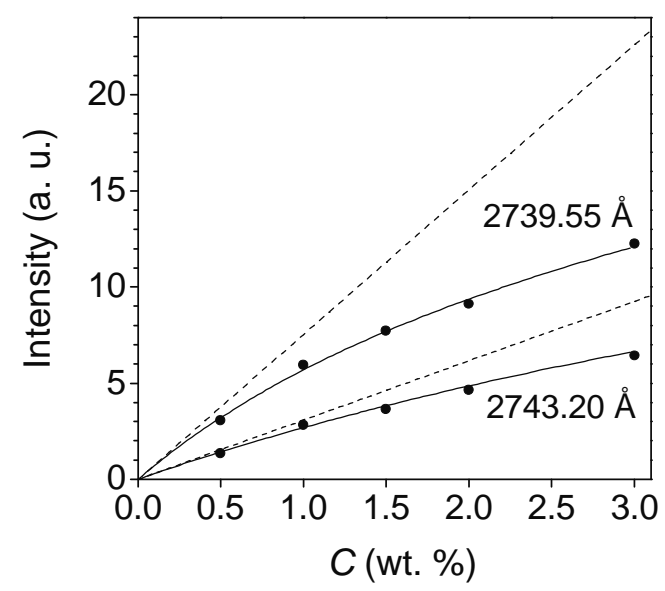

Figure 5. Experimental and calculated curves of growth of Fe II lines. The linear asymptotes for low concentrations are shown as dashed lines.

\section{Measurements of Stark widths}

\subsection{Selection of the iron concentration in the sample}

The characterization of the laser-induced plasma described in the previous section has been used to determine the iron concentration in the sample required to maintain a low self-absorption of the Fe II lines in the measurement of Stark widths. The procedure followed is described next for some lines taken as examples, making use of table 1. For each line, the multiplet and atomic data, including level energies and degeneracies, transition probability and oscillator strength are indicated. From the atomic data and the plasma temperature, the coefficient $k_{t}$ is calculated [11]. This coefficient, shown in table 1 for a temperature of $14500 \mathrm{~K}$, may be defined as the part of the effective absorption coefficient that may be calculated from the transition parameters and the temperature. The use of $k_{t}$ is advantageous because, on one side, the intensity of a line in the optically thin limit is proportional to the product of $k_{t}$ times the slowly-varying Planck blackbody distribution. On the other side, for a given damping constant, $k_{t}$ determines the selfabsorption of the line. Specifically, the concentration $C_{\text {int }}$ that defines the intersection of the low and high optical depth asymptotes of the curve of growth is inversely proportional to $k_{t}$ [12]. As we can see in table 1 , lines of very different intensities, with $k_{t}$ values in a wide range, have been included in the study. For each line, the calculation of the curve of growth using the plasma parameters determined previously allows obtaining the concentration $C_{10 \%}$ for $10 \%$ selfabsorption, i.e., leading to a line intensity $10 \%$ lower than the intensity in the optically thin limit. In this estimation, the value of the Stark width has been taken as $0.05 \AA$ for all the lines, according to previous measurements [11] and initial estimations. As seen in table 1, the value of $C_{10 \%}$ is in the range $0.29-22 \mathrm{wt}$. \% for the lines selected. The last column of the table shows the concentration $C$ finally used in the measurement of the Stark width, chosen as the nearest concentration to $C_{10 \%}$ available in the samples. 
Table 1. Atomic data of some of the lines studied, $k_{t}$ value, concentration $C_{10 \%}$ for $10 \%$ self-absorption and iron concentration $C$ used in measurements.

\begin{tabular}{|c|c|c|c|c|c|c|c|c|c|c|}
\hline$\lambda(\AA)$ & Multiplet & $\begin{array}{l}E_{i} \\
(\mathrm{eV})\end{array}$ & $\begin{array}{l}E_{k} \\
(\mathrm{eV})\end{array}$ & $g_{i}$ & $g_{k}$ & $\begin{array}{l}A_{k i} \\
\left(10^{8} \mathrm{~s}^{-1}\right)\end{array}$ & $f_{i k}$ & $\begin{array}{l}k_{t}(14500 \mathrm{~K}) \\
\left(10^{-30} \mathrm{~m}^{3}\right)\end{array}$ & $\begin{array}{l}C_{10 \%} \\
\text { (wt. \%) }\end{array}$ & $\begin{array}{l}C \\
\text { (wt. \%) }\end{array}$ \\
\hline 2739.55 & $a^{4} \mathrm{D}-z^{4} \mathrm{D}^{\circ}$ & 0.99 & 5.51 & 8 & 8 & 2.21 & 0.249 & 5.96 & 0.29 & 0.5 \\
\hline 2743.20 & $a^{4} \mathrm{D}-z^{4} \mathrm{~F}^{\circ}$ & 1.10 & 5.62 & 2 & 4 & 1.97 & 0.445 & 2.44 & 0.73 & 0.5 \\
\hline 2753.29 & $b^{2} \mathrm{H}-z^{2} \mathrm{I}^{\circ}$ & 3.27 & 7.77 & 10 & 12 & 1.89 & 0.258 & 1.25 & 1.41 & 1.5 \\
\hline 2755.74 & $a^{4} \mathrm{D}-z^{4} \mathrm{~F}^{\circ}$ & 0.99 & 5.48 & 8 & 10 & 2.15 & 0.306 & 6.24 & 0.27 & 0.5 \\
\hline 2761.81 & $a^{4} \mathrm{D}-z^{4} \mathrm{D}^{\circ}$ & 1.10 & 5.58 & 2 & 4 & 0.138 & 0.0315 & 0.17 & 10.1 & 10 \\
\hline 2875.35 & $a^{2} \mathrm{~F}-z^{2} \mathrm{G}^{\circ}$ & 3.39 & 7.70 & 8 & 10 & 0.135 & 0.021 & 0.081 & 22.0 & 25 \\
\hline
\end{tabular}

\subsection{Measurement and fitting of the line profiles}

The spectra of the Fe II lines of interest were measured using in each case the sample with an iron concentration determined as described in the previous subsection. The lines were fitted to Voigt profiles to determine the Lorentzian widths. Figure 6 shows the spectra at $1.3 \mu$ s of two Fe II lines measured with iron concentrations of $0.5 \mathrm{wt} . \%$ (figure $6 \mathrm{a}$ ) and $1.5 \mathrm{wt} \%$ (figure $6 \mathrm{~b}$ ). In each plot, the fitting to the Voigt profile is shown for the line whose Stark width is determined with this concentration. By comparing the ratio of the areas of the lines, we appreciate the higher effect of self-absorption in figure $6 \mathrm{~b}$, where the ratio $(4.0 \pm 0.1)$ is smaller than that deduced from figure $6 \mathrm{a}(4.74 \pm 0.06)$, the latter corresponding to a lower concentration. Also, the slightly higher broadening of the line at $2755.75 \AA$ in the spectrum of figure $6 \mathrm{~b}$ may be noticed. As discussed previously, the concentration used for each line is chosen so that effect of self-absorption in the area is limited to about $10 \%$.
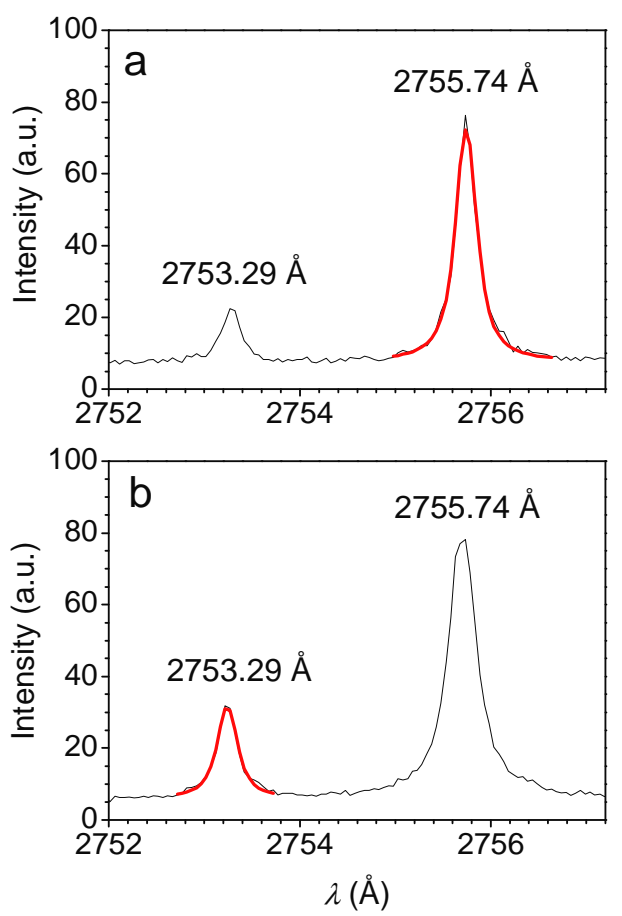

Figure 6. Spectra of Fe II lines measured using the Fe-Cu sample with 0.5 wt. \% (a) and 1.5 wt. \% (b). Each plot shows the fitting of the line whose Stark width is measured with this concentration. 
The grating of 3600 lines $/ \mathrm{mm}$ and a slit width of $20 \mu \mathrm{m}$ were used in the measurements of the Fe II spectra. The instrumental width, determined from the line spectra emitted by a lowpressure mercury lamp, was $0.10 \AA$. The Doppler width is estimated for a temperature of 14000 $\mathrm{K}$ as $0.03 \AA$, so the combination of the instrumental and Doppler widths leads to a total Gaussian width of $0.11 \AA$. In order to assure the accuracy of the Stark widths, only the data corresponding to instants at which the Lorentzian width is higher or similar to the Gaussian width have been used in the measurements. Therefore, the time window centered at $3.6 \mu$ s has not been included, and the Stark widths have been determined from $0.84 \mu$ s to $2.5 \mu$ s, at electron densities in the range (1.6-7.3) $\times 10^{17} \mathrm{~cm}^{-3}$, with typical Lorentzian width values in the range 0.07-0.4 $\AA$. The corresponding range of plasma temperatures was $12900-15200 \mathrm{~K}$. This range was too small to detect the weak dependence of the Stark widths on temperature, taking into account the statistical error of both magnitudes. From the experimental results, we have found proportionality between the Stark widths determined at each instant of the plasma evolution and the corresponding electron density. A typical plot showing this proportionality is presented in figure 7, where the error bars represent the statistical errors. The final value given in this work is the slope of a linear fitting of this plot with zero intercept, this value corresponding to the Stark width at an electron density of $10^{17} \mathrm{~cm}^{-3}$. The statistical error is taken as the standard deviation of the slope, which is in the range 1-4 \%.

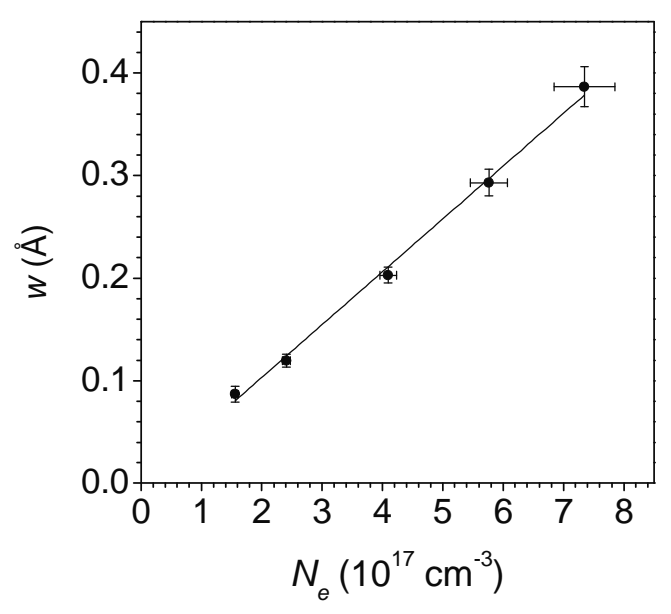

Figure 7. Stark width (FWHM) of the Fe II line at 2743.20 ̊ as a function of the electron density.

\subsection{Estimation of the error due to self-absorption}

The curve-of-growth methodology allows estimating the remaining error of the Stark widths due to self-absorption. This study has been carried out for the two lines of different intensity at $2743.20 \AA$ and $2739.55 \AA$ (see the $k_{t}$ values in table 1) whose curves of growth are plotted in figure 5. In this figure, the linear asymptotes of the curves of growth are also shown as dashed lines. This allows appreciating the departure of the area of the lines from the optically thin limit that, for a given concentration, is higher for the most intense line. In this work, we are interested in the over-estimation of the Stark widths. Figure 8 shows in solid symbols the experimental results for the Stark widths, determined as described in the previous subsection, as a function of the iron concentration in the sample. As expected, the over-estimated width increases with the concentration in the sample and, for a given concentration, is higher for the more intense line. 
The curves of growth of figure 5 provide the over-estimation of the area; from this value, the over-estimation of the Stark width has been determined. The Stark widths of the two lines corrected from self-absorption have then been obtained, and are shown in figure 8 as open symbols. As can be seen, for the line of moderate intensity at $2743.20 \AA$, the corrected values are similar for all concentrations. This is an indication that the method provides a quite accurate correction of self-absorption when the optical depth is relatively small, as is the case of this line for concentrations up to $3 \mathrm{wt} . \%$. The average of the corrected values is indicated in the figure as a solid line. Taking this average as the true Stark width, the error of the measurement carried out with the sample of $0.5 \mathrm{wt}$. $\%$ amounts to $6 \%$ for this line. In the case of the more intense line at $2739.55 \AA$, the correction works well up to a concentration of 1.5 wt. \%. For higher concentrations, the corrected Stark widths deviate towards small values. This deviation is explained by the failure of the simple model used, that considers an homogeneous laser-induced plasma. As has been described previously [16], the model fails for intense lines at high concentrations, i.e., for high optical depths, because in this case a significant part of the emission comes from the plasma borders, where strong spatial gradients of the plasma parameters are present. If we take for this line the average of the corrected values up to $1.5 \mathrm{wt}$. $\%$ as the true Stark width (dashed line) the error due to self-absorption for the $0.5 \mathrm{wt}$. \% concentration used in the measurement is $10 \%$.

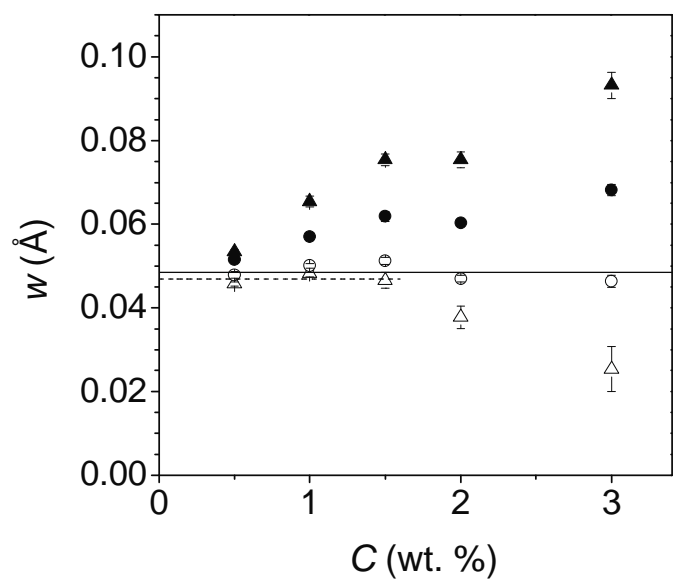

Figure 8. Stark widths of Fe II lines as a function of the iron concentration in the sample, measured for the line at $2743.20 \AA$ (solid circles) and the more intense line at $2739.55 \AA$ (solid triangles). The corresponding values corrected from self-absorption are shown as open symbols.

\subsection{Results for Stark widths}

The experimental results obtained in this work for the Stark widths of Fe II lines are presented in table 2. For each line, the transition, multiplet number, multiplet, and wavelength are indicated. The total experimental error has been estimated as $14 \%$ by the combination of the statistical error (lower than 4\%), the error due to self-absorption (lower than $10 \%$ ) and the error of the electron density (11\%). For three resonance lines, our results are compared to the values measured by Purić et al. [1] using a linear arc discharge plasma source at an electron density range of $(1.06-1.27) \times 10^{17} \mathrm{~cm}^{-3}$ and an electron temperature from $28000 \mathrm{~K}$ to $29000 \mathrm{~K}$. For comparison, the results of [1] have been normalized to an electron density of $10^{17} \mathrm{~cm}^{-3}$. If we do not consider the high difference of temperatures between the two sources, our results are almost compatible with those in [1] for two of the lines, whereas a higher difference is obtained for the 
line at $2611.87 \AA$. For four lines, the Stark widths were obtained in a previous experiment of our group [11], using a laser-induced plasma in similar conditions, but with a different reference line to determine the electron density and a higher instrumental width. The present results complete and increase the accuracy of our previous measurements. Finally, the theoretical results by Dimitrijević [2], obtained for a temperature of $28000 \mathrm{~K}$ are, when normalized to an electron density of $10^{17} \mathrm{~cm}^{-3}$, higher than our measurements in about $60 \%$.

Table 2. Stark width (FWHM) at electron density $10^{17} \mathrm{~cm}^{-3}$ of Fe II spectral lines.

\begin{tabular}{|c|c|c|c|c|c|c|c|}
\hline \multirow[b]{3}{*}{ Transition } & \multirow[b]{3}{*}{$\mathrm{N}^{\circ}$} & \multirow[b]{3}{*}{ Multiplet } & \multirow[b]{3}{*}{$\lambda(\AA)$} & \multicolumn{4}{|c|}{$w(\AA)$} \\
\hline & & & & \multicolumn{3}{|c|}{$\operatorname{Exp}$} & \multirow{2}{*}{$\begin{array}{l}\mathrm{Th} \\
{[2]}\end{array}$} \\
\hline & & & & This work $^{\mathrm{a}}$ & {$[1]$} & [11] & \\
\hline \multirow[t]{12}{*}{$3 d^{6}\left({ }^{5} D\right) 4 s-3 d^{6}\left({ }^{5} D\right) 4 p$} & 1 & $a^{6} \mathrm{D}-z^{6} \mathrm{D}^{\circ}$ & 2611.87 & 0.0368 & 0.068 & & 0.062 \\
\hline & & & 2607.09 & 0.0394 & 0.046 & & 0.061 \\
\hline & & & 2613.82 & 0.0384 & 0.042 & & 0.062 \\
\hline & & & 2628.29 & 0.0396 & & & \\
\hline & 32 & $a^{4} \mathrm{D}-z^{6} \mathrm{~F}^{\circ}$ & 2926.59 & 0.048 & & & \\
\hline & 34 & $a^{4} \mathrm{D}-z^{4} \mathrm{~F}^{\circ}$ & 2755.74 & 0.0540 & & & \\
\hline & & & 2746.48 & 0.054 & & & \\
\hline & & & 2743.20 & 0.0515 & & & \\
\hline & & & 2730.73 & 0.050 & & 0.05 & \\
\hline & 35 & $a^{4} \mathrm{D}-z^{4} \mathrm{D}^{\circ}$ & 2739.55 & 0.0534 & & 0.07 & \\
\hline & & & 2746.98 & 0.056 & & & \\
\hline & & & 2761.81 & 0.053 & & & \\
\hline $3 d^{6}\left({ }^{3} \mathrm{P} 2\right) 4 s-3 d^{6}\left({ }^{3} \mathrm{P} 2\right) 4 p$ & 140 & $b^{2} \mathrm{P}-z^{2} \mathrm{D}^{\circ}$ & 2831.56 & 0.049 & & & \\
\hline $3 d^{6}\left({ }^{3} H\right) 4 s-3 d^{6}\left({ }^{3} F 2\right) 4 p$ & 151 & $b^{2} \mathrm{H}-y^{4} \mathrm{~F}^{\circ}$ & 2799.30 & 0.054 & & & \\
\hline \multirow[t]{3}{*}{$3 d^{6}\left({ }^{3} H\right) 4 s-3 d^{6}\left({ }^{3} H\right) 4 p$} & 152 & $b^{2} \mathrm{H}-z^{2} \mathrm{G}^{\circ}$ & 2783.69 & 0.0504 & & & \\
\hline & & & 2779.30 & 0.0532 & & & \\
\hline & 153 & $b^{2} \mathrm{H}-z^{2} \mathrm{I}^{\circ}$ & 2753.29 & 0.0532 & & 0.06 & \\
\hline \multirow[t]{2}{*}{$3 d^{6}\left({ }^{3} F 2\right) 4 s-3 d^{6}\left({ }^{3} F 2\right) 4 p$} & 169 & $a^{2} \mathrm{~F}-z^{2} \mathrm{G}^{\circ}$ & 2875.35 & 0.0489 & & & \\
\hline & 175 & $a^{2} \mathrm{~F}-y^{2} \mathrm{G}^{\circ}$ & 2664.66 & 0.0479 & & & \\
\hline \multirow[t]{2}{*}{$3 d^{6}\left({ }^{3} G\right) 4 s-3 d^{6}\left({ }^{3} G\right) 4 p$} & 193 & $b^{2} \mathrm{G}-y^{2} \mathrm{H}^{\circ}$ & 2692.60 & 0.0501 & & 0.06 & \\
\hline & & & 2684.75 & 0.048 & & & \\
\hline
\end{tabular}

${ }^{a}$ Experimental error $14 \%$. The temperature range is $12900-15200 \mathrm{~K}$.

\section{Conclusions}

We report measurements of Stark widths of Fe II lines with wavelengths in the range 260-300 $\mathrm{nm}$, carried out by laser-induced plasma spectroscopy. The measurements presented, practically new in the literature, are important for different fields, particularly in astrophysics. The method used to control self-absorption, based in the selection of the iron concentration in the sample, has allowed including intense and weak lines belonging to 11 multiplets, some of them with high upper level energies. 


\section{References}

[1] Purić J, Djeniže S, Srećković A, Bukvić S, Pivalica S and Labat J 1993 Astron. Astrophys. Suppl. Ser. 102607

[2] Dimitrijević M S 1995 Astron. Astrophys. Suppl. Ser. 111565

[3] Fuhr J R and Wiese W L 2006 J. Phys. Chem. Ref. Data 351669

[4] Lesage A 2009 New Astron. Rev. 52471

[5] Mayo R, Ortiz M and Plaza M 2008 J. Phys. B: At. Mol. Opt. Phys. 41095702

[6] Mayo R and Ortiz M 2008 J. Phys. B: At. Mol. Opt. Phys. 41225702

[7] Alonso-Medina A and Colón C 2006 Astrophys. J. 6721286

[8] Colón C and Alonso-Medina A 2006 Spectrochim. Acta Part B 61856

[9] Bredice F, Borges F O, Sobral H, Villagran-Muniz M, Di Rocco H O, Cristoforetti G, Legnaioli S, Palleschi V, Salvetti A and Tognoni E 2007 Spectrochim. Acta Part B 621237

[10] Konjević N, Ivković M and Jovićević S 2010 Spectrochim. Acta Part B 65593

[11] Aragón C, Peñalba F and Aguilera J A 2005 Spectrochim. Acta Part B 60879

[12] Aguilera J A and Aragón C 2008 Spectrochim. Acta Part B 63784

[13] Gigosos M A and Cardeñoso V 1996 J. Phys. B: At. Mol. Opt. Phys. 294795

[14] Aragón and Aguilera J A 2010 Spectrochim. Acta Part B 65395

[15] McWhirter R W P 1965 Plasma Diagnostic Techniques (Pure and Applied Physics vol 21) eds R H Huddlestone and S L Leonard (New York: Academic Press) pp 201-264

[16] Aguilera J A and Aragón C 2004 Spectrochim. Acta Part B 591861

[17] Aguilera J A, Bengoechea J and Aragón C 2003 Spectrochim. Acta Part B 58221 